\title{
Detection Using Intermittent Observations for Passive Wireless Sensors
}

\author{
Ashraf Tantawy, Xenofon Koutsoukos, and Gautam Biswas
}

\begin{abstract}
Passive wireless sensors have emerged as a new technology to measure multiple phenomena in our daily life. Passive sensors require no power source, and therefore their application domains are numerous, including health care, infrastructure protection, and national security. The deployment of passive wireless sensors and their readers has changed how detection needs to be performed. Passive sensors cannot preprocess the measurements as they have limited computational power. Therefore, no local decision is taken. Also, the reader polls the information from multiple sensors at the same time, and this causes collisions and hence packet drops and delays. Detectors designed without considering the properties of the communication channel have degraded performance. Therefore, analysis is required to quantify the degradation and take the necessary remedy action. In this paper, we study the effect of sensor-reader channel imperfection on the local detection performance of the reader, assuming no data pre-processing at the passive sensor. We consider the case of a single sensor-reader communication over a Bernoulli communication channel. We formulate the detector performance and compare with the ideal case. We present the problem of DC level detection in White Gaussian Noise, as a case study. Finally, we propose a heuristic approach to restore the original detector performance working with non-ideal channel, with the cost of increasing the delay for detection.
\end{abstract}

\section{INTRODUCTION}

In classical detection theory, statistical hypothesis testing is applied to detect noisy signals. The main problem is to design the optimal detector (according to some pre-defined criteria) that distinguishes between two or more hypotheses (sometimes referred to as phenomena or state of nature) given noisy observations. This problem has been studied extensively, and different detectors have been proposed [6]. Examples are the Neyman-Pearson (NP) detector and the minimum Bayes risk detector, with its special case the Maximum Likelihood (ML) detector [10]. Classical detection theory will be referred to as centralized detection hereafter.

The emergence of distributed radar has given another perspective for the detection problem, namely Decentralized Detection (DD). DD has been an active area of research during the 1980s and early 1990s. In DD, multiple sensors relay information (after pre-processing) to a fusion center, and the problem is to design both the optimal local decision rules and the fusion rule to detect events as accurately as possible [1]. The recent emergence of Wireless Sensor Networks (WSNs) has added to the challenge of detection the issue of channel imperfection, which causes delays and missed

The authors are with the Institute for Software Integrated Systems and the Department of Electrical Engineering and Computer Science, Vanderbilt University, Nashville, TN, 37235, USA (email: ashraf.tantawy@vanderbilt.edu; xenofon.koutsoukos@vanderbilt.edu; gautam.biswas@vanderbilt.edu). observations. There is some recent work that discusses the effect of non-ideal channels on the design of decentralized detectors [2], [4]. However, the main assumption is that the sensor nodes have sufficient computational power to preprocess the observations and take a decision. This assumption is no longer valid in the case of passive wireless sensors.

In the last few years, passive wireless sensors have emerged as a new technology for sensors that does not require any power source. The basic idea is to use a powered reader to poll the data from different passive sensors. The passive sensor uses the incident power from the reader to energize its local circuitry. A variety of ways exist for the sensor to modulate the reader incident wave and relay its information back to the reader. The most popular example for this architecture is RFID technology [7].

Motivated by RFID technology, passive wireless sensors have been developed to measure multiple phenomena in our daily life. In the environmental monitoring domain, passive wireless sensors that detect bio-hazardous materials are explained in [18]. An example of a passive wireless sensor for measuring temperature, stress, strain, acceleration and displacement using Surface Acoustic Wave (SAW) transducer is described in [8]. In the health care domain, a passive strain-sensor technology for the measurement of small strains on bones or fixation systems in the human body is presented in [16]. In the automotive industry, passive wireless strain monitoring of car tires is described in [9].

The application domains for passive sensors has increased considerably in the last few years. This is due to the fact that many applications require the deployment of sensors over long periods of time, often years, without the possibility of battery replacement or recharging. Domains of applications include, but are not limited to, health care, infrastructure protection, food transportation, national security, automotive industry, environmental monitoring, disaster forecast, and industrial automation [19]. The technology also advances everyday giving rise to smaller sensors, longer reading distances, and better communication protocols.

The introduction of passive wireless sensors as a replacement for active sensors has changed how detection needs to be performed. The main change comes from the fact that a passive sensor has limited computational power and memory, and therefore, it cannot pre-process the measurements before transmission to the reader. In addition, because of the limited power supplied to the sensor, the reflected signal has very small power, which makes detection a much more challenging task especially if the noise power is large. Finally, in a passive WSN architecture, the reader polls the information from multiple sensors at the same time, giving 
rise to collisions and hence packet drops and delays.

In this paper, we study the effect of sensor-reader channel imperfection on the local detection performance of the reader. We use the terms reader and detector interchangeably in this paper, since the detector has the additional role of polling the sensor data. We formulate the detector performance, working with a Bernoulli communication channel, and present a case study for the problem of DC level detection in White Gaussian Noise (WGN). We compare analytically the detection performance with perfect communication channel against a Bernoulli communication channel. The analytical results are verified by Monte Carlo simulation studies. We also propose an adaptive detector architecture to restore the original detector performance working with a non-ideal channel.

The rest of the paper is organized as follows: In Section III, we present the problem formulation for detection with reader/passive sensor pair, communicating over a Bernoulli communication channel. We formulate the detection performance in section IV, and present a case study in Section V. In section VI, we propose an adaptive detector architecture to restore the original detector performance working with non-ideal channel. Finally, we conclude the work with future research directions in section VII.

\section{RELATED WORK}

Classical detection theory, with particular attention to signal processing applications, is discussed in [5], [6], [10]. The research on decentralized detection is largely attributed to the seminal work of Tenney and Sandell [14]. The optimal decision rules for the local nodes and the fusion center are derived under various problem settings and different optimality criteria. Comprehensive surveys in this area can be found in [1], [3] and the references therein.

The inclusion of the resource constraints imposed by WSNs has been reported in recent work. Kalman filter performance with intermittent observations is studied in [11]. Fading channel layer is incorporated in the classical parallel fusion structure in [2]. The optimality of the LikelihoodRatio test for local sensor decision rules in the presence of non-ideal channels is proved in [4]. Distributed decision fusion with networking delays and channel errors is considered in [15]. Comprehensive surveys on the early results in this emerging field can be found in [1], [3], [17].

We have considered Maximum Likelihood detection with intermittent observations in [13], where the optimal decision rule is derived and performance analysis is carried out. Further, we have considered the performance of the Z-test with intermittent observations in [12]. In this paper, we formulate the detection problem with intermittent observations, according to NP criterion.

\section{PROBLEM FORMULATION}

We consider the problem of a single passive sensor to reader (detector) communication over a non-ideal channel. Figure 1 illustrates an abstract representation for the problem. The state of nature is sensed by a passive wireless sensor, and the observation is transmitted over a Bernoulli wireless channel to the local detector (reader). Information relaying happens only when the reader polls the sensor data. The objective is twofold: analyzing the detection performance assuming a Bernoulli channel, and restoring the original detector performance.

Information loss is expected with non-ideal channel. Therefore, the first objective, performance analysis, is to quantify the performance degradation in the detector. The later objective, performance restoration, is achieved by estimating the channel drop rate, and using a pre-constructed Delay-Drop Rate curve, to increase the number of samples.

We consider the binary hypothesis testing problem where the state of nature is represented by one of two hypotheses, $\mathcal{H}_{0}$ and $\mathcal{H}_{1}$. An observation vector, $\mathbf{x}$, is used to statistically validate one of the two hypotheses. The decision is governed by the NP criterion for maximizing the probability of detection, $P_{D}$, given the probability of false alarm, $P_{F A}$. The optimal test is the likelihood ratio test:

$$
l(\mathbf{x}) \underset{\mathcal{H}_{0}}{\stackrel{\mathcal{H}_{1}}{\gtrless}} \gamma
$$

The detector performance is given by:

$$
\begin{aligned}
P_{F A} & =P\left(l(\mathbf{x})>\gamma ; \mathcal{H}_{0}\right)=\int_{\gamma}^{\infty} p\left(l ; \mathcal{H}_{0}\right) d l \\
P_{D} & =P\left(l(\mathbf{x})>\gamma ; \mathcal{H}_{1}\right)=\int_{\gamma}^{\infty} p\left(l ; \mathcal{H}_{1}\right) d l
\end{aligned}
$$

The relationship between $P_{D}$ and $P_{F A}$ is known as the Receiver Operating Characteristics (ROC) curve.

In the centralized detection framework, the existence of the unreliable channel is ignored, and, therefore, perfect knowledge about the observations is assumed (a classical example is a radar system). For the work presented in this paper, we assume a Bernoulli channel with a probability of failure, $\lambda$, for dropped observations. Therefore, the channel is characterized by a random variable $C$ with a Probability Mass Function (PMF):

$$
\begin{aligned}
& P_{C}(c=0)=\lambda \\
& P_{C}(c=1)=1-\lambda
\end{aligned}
$$

Because of loss of information from the dropped observations, degradation in the detector performance is expected. We analyze the performance of the detector for the nonideal channel, and compare with the ideal channel case, by comparing the ROC curves in both cases. Also, in many applications, it is required to restore the original performance under degraded conditions. Since in all detection problems there is always a trade-off between performance and delay for detection, we quantify the extra delay required for performance restoration, by defining the relationship between the extra delay needed and the channel drop rate $\lambda$. 


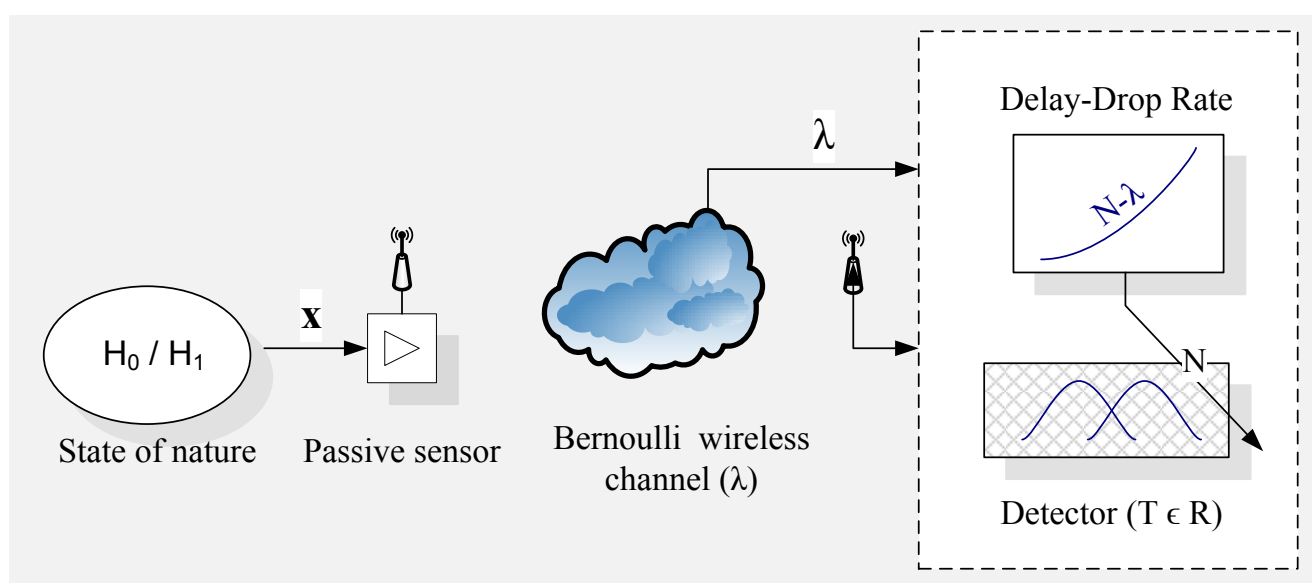

Fig. 1. Adaptive NP detection for wireless passive sensor networks

\section{NP DETECTION PERFORMANCE WITH BERNOULLI CHANNEL}

In the case of an ideal channel between the reader and passive sensor, the detector is designed based on a number of observations $N$, received in a time period $T_{p}$, to satisfy a required operating point $\left(P_{F A}, P_{D}\right)$. However, in the case of a Bernoulli communication channel, not all observations are received at the reader. Therefore, in a time period $T_{p}$, the number of observations received $k \leq N$. The probability of receiving $k$ out of $N$ observations, in $T_{p}$, is given by:

$$
P(K=k)=\sum_{k=0}^{N}\left(\begin{array}{l}
N \\
k
\end{array}\right)(1-\lambda)^{k} \lambda^{(N-k)}
$$

Therefore, with every realization of $K$, the detector performance pair $\left(P_{F A}^{k}, P_{D}^{k}\right)$ is no longer a deterministic quantity, but represents a pair of random variables. The performance of the NP detector in this case can be described by the pair of expected values $\left(E\left[P_{F A}^{k}\right], E\left[P_{D}^{k}\right]\right)$ :

$$
\begin{aligned}
E\left[P_{F A}^{k}\right] & =\sum_{k=0}^{N}\left(\begin{array}{l}
N \\
k
\end{array}\right)(1-\lambda)^{k} \lambda^{(N-k)} P_{F A}^{k} \\
E\left[P_{D}^{k}\right] & =\sum_{k=0}^{N}\left(\begin{array}{l}
N \\
k
\end{array}\right)(1-\lambda)^{k} \lambda^{(N-k)} P_{D}^{k}
\end{aligned}
$$

The relationship between $E\left[P_{D}^{k}\right]$ and $E\left[P_{F A^{k}}\right]$ represents the new ROC curve, to be compared with the ROC curve for the detector with ideal channel. It should be noted that Equations (3) and (4) reduce to Equations (1) and (2), respectively, when $\lambda=0$. Section V compares the ROC curves for the classical DC level in WGN detection problem.

To overcome the performance degradation, one way is to increase the number of samples $N$. This can be done by increasing the decision time period $T_{p}$. Therefore, this approach enhances the performance, with the penalty of increasing the delay for detection. Section VI illustrates the approach for performance restoration.

\section{NP DETECTION-DC LEVEL IN WGN}

\section{A. DETECTION PERFORMANCE}

The problem of detecting a known DC level in WGN is formulated as:

$$
\begin{array}{ll}
\mathcal{H}_{0} & : \quad x[n]=\omega[n] \\
\mathcal{H}_{1} & : \quad x[n]=A+\omega[n]
\end{array}
$$

where $n=0,1, \ldots, N-1, x[n]$ represents the sample sequence, $A$ is the known DC level to be detected, and $w[n]$ represents WGN with zero mean and variance $\sigma^{2}$. If $A>0$ it can be shown that the NP detector reduces to testing the mean of the observations against a threshold value that is defined by the desired $P_{F A}$ [6]. The detector performance is given by:

$$
\begin{aligned}
P_{F A} & =Q\left(\frac{\gamma}{\sqrt{\sigma^{2} / N}}\right) \\
P_{D} & =Q\left(\frac{\gamma-A}{\sqrt{\sigma^{2} / N}}\right) \\
P_{D} & =Q\left(Q^{-1}\left(P_{F A}\right)-\sqrt{\frac{N A^{2}}{\sigma^{2}}}\right)
\end{aligned}
$$

where $Q($.$) is the error function defined by:$

$$
Q(x)=\int_{x}^{\infty} \frac{1}{\sqrt{2 \pi}} e^{-\frac{1}{2} t^{2}} d t
$$

From Equation (7), the detection performance increases with $\left(N A^{2} / \sigma^{2}\right)$. This quantity is referred to as the deflection coefficient, $d^{2}$ [6]. Therefore, to get better detection performance either the signal to noise ratio has to be high (which is usually not under user control) or $N$ should be increased, which corresponds to a delay in detection.

Now we assume a non-ideal Bernoulli channel. We further assume that the detector was designed based on the assumption of ideal channel, and that the decision rule and threshold, $\gamma$, are fixed. Therefore, at the end of every decision period $T_{p}$, the detector compares the sample mean to the original threshold $\gamma$, irrespective of the number of samples 
received. Accordingly, it can be easily shown that $P_{F A}^{k}$ and $P_{D}^{k}$ are obtained from Equations (5) and (6), respectively, by replacing $N$ by $k$. Using Equations (3) and (4), the detector performance is expressed as:

$$
\begin{gathered}
E\left[P_{F A}\right]=\sum_{k=0}^{N}\left(\begin{array}{l}
N \\
k
\end{array}\right)(1-\lambda)^{k} \lambda^{(N-k)} Q\left(\frac{\gamma}{\sqrt{\sigma^{2} / k}}\right) \\
E\left[P_{D}\right]=\sum_{k=0}^{N}\left(\begin{array}{l}
N \\
k
\end{array}\right)(1-\lambda)^{k} \lambda^{(N-k)} Q\left(\frac{\gamma-A}{\sqrt{\sigma^{2} / k}}\right)
\end{gathered}
$$

Example 1. Assume it is required to design an NP detector for DC level in WGN, with parameters $A=0.2, \sigma=1, N=$ 100 , and a constraint $P_{F A}=0.05$. From Equations (5) and (6) we get $\gamma=0.1645, P_{D}=0.6387$. Therefore, the detector operating point is $(0.05,0.6387)$.

Now the detector is designed with $\gamma=0.1645$. Assuming a non-ideal, Bernoulli channel with packet drop rate estimated by $\lambda=0.2$, and substituting in Equations (8) and (9) we get $E\left[P_{F A}\right]=0.0708, E\left[P_{D}\right]=0.6245$. Therefore, the new detector operating point is $(0.0708,0.6245)$, so the detector is working with higher probability of false alarm and lower probability of detection than the ideal channel case.

\section{B. ROC CURVE}

Equations (8) and (9) represent the ROC curve for the NP detector with intermittent observations. Using detector parameters in Table I, the ROC curve is plotted in Figure 2 for different values of $\lambda$, including the ideal case $(\lambda=0)$. The performance degrades with increasing values of channel drop rate, for all values of the detector threshold. Therefore, the degradation is irrecoverable using the original detector sample size. This is intuitive since increasing the channel drop rate represents a loss of information at the reader, therefore the reader has to decide on the state of nature with a subset of the original information.

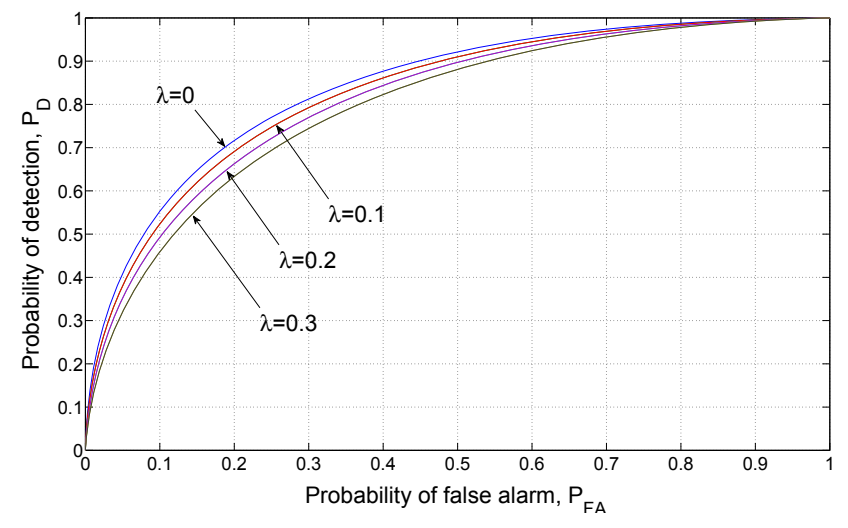

Fig. 2. ROC curve-NP detector with intermittent observations

To verify the analytical results, a Monte Carlo simulation experiment is conducted. Samples are generated from two different Gaussian distributions (corresponding to the two hypotheses), and a Bernoulli channel is introduced in the signal path to the detector. The detector calculates a running
TABLE I

Detector Parameters-RoC Curve

\begin{tabular}{|c||c|c|c|c|c|c|}
\hline Parameter & $A$ & $\sigma$ & $N$ & $P_{F A}$ & $P_{D}$ & $\gamma$ \\
\hline Value & 0.2 & 1 & 50 & 0.05 & 0.4088 & 0.2326 \\
\hline
\end{tabular}

average, and it ignores dropped observations. The detector compares the running average to a threshold value that varies from $-\infty$ to $\infty$, to generate the complete ROC curve. For each value of the detector threshold, 5000 Monte Carlo trials were performed to get accurate values for $P_{F A}$ and $P_{D}$. Table II lists the parameters used in the simulation experiment.

TABLE II

Detector Parameters-Monte Carlo Simulation

\begin{tabular}{|c||c|c|c|c|c|}
\hline Parameter & $A$ & $\sigma$ & $N$ & $\lambda$ & $\gamma$ \\
\hline Value & $0,0.2$ & 1 & 100 & $0,0.1$ & $(-\infty, \infty)$ \\
\hline
\end{tabular}

Figure 3 shows the theoretical ROC curve (Equations (8) and (9)) versus the ROC curve obtained from Monte Carlo simulation, for $\lambda=0$ and $\lambda=0.1$. As illustrated in the figure, the two ROC curves for each value of $\lambda$ are almost indistinguishable.

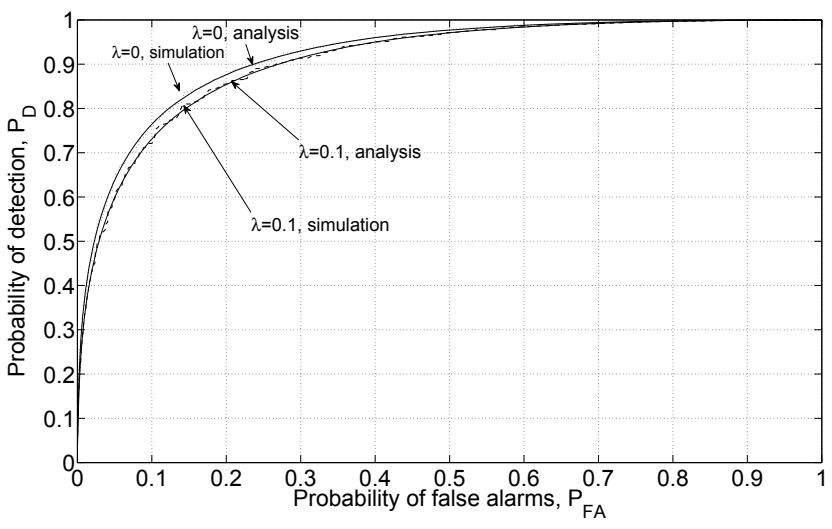

Fig. 3. ROC curve-NP detector with intermittent observations-Monte Carlo simulations

The degradation in the performance is clear from the new ROC curve. For a fixed $P_{F A}$, the original $P_{D}$ cannot be obtained using the same number of samples. One way to restore the original performance is to increase the number of samples. By doing that, we obtain different ROC curves that approach the original one. Figure 4 illustrates this fact, where different curves correspond to different number of samples $N$. For the detector with parameters as in Table $\mathrm{I}(\lambda=0.2)$, the number of samples required to obtain the same original ROC curve is $N=63$, compared to the original case of $N=50$ (see section VI for numerical results). So, the price paid for the channel imperfection is an $28 \%$ increase of the detection delay.

\section{ADAPTIVE DETECTOR DESIGN}

To compensate for the degradation in the performance of the detector, one solution is to increase the number of 


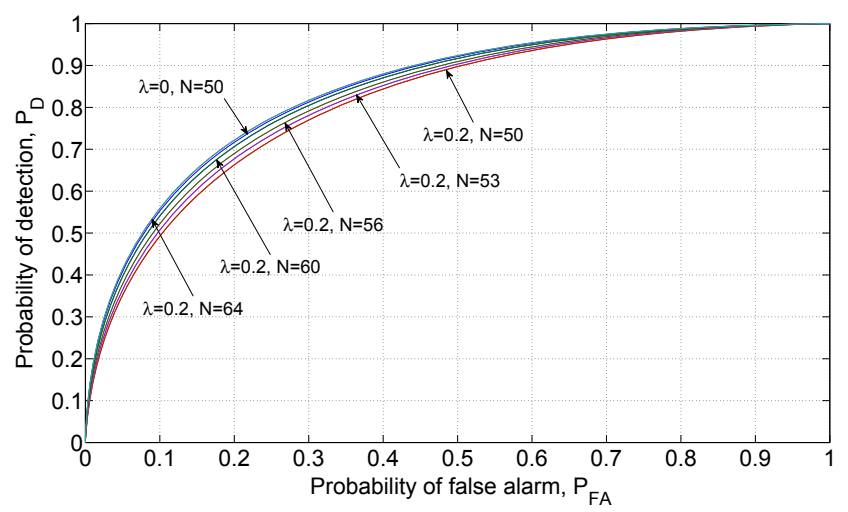

Fig. 4. ROC curve-NP detector with intermittent observations, increasing samples

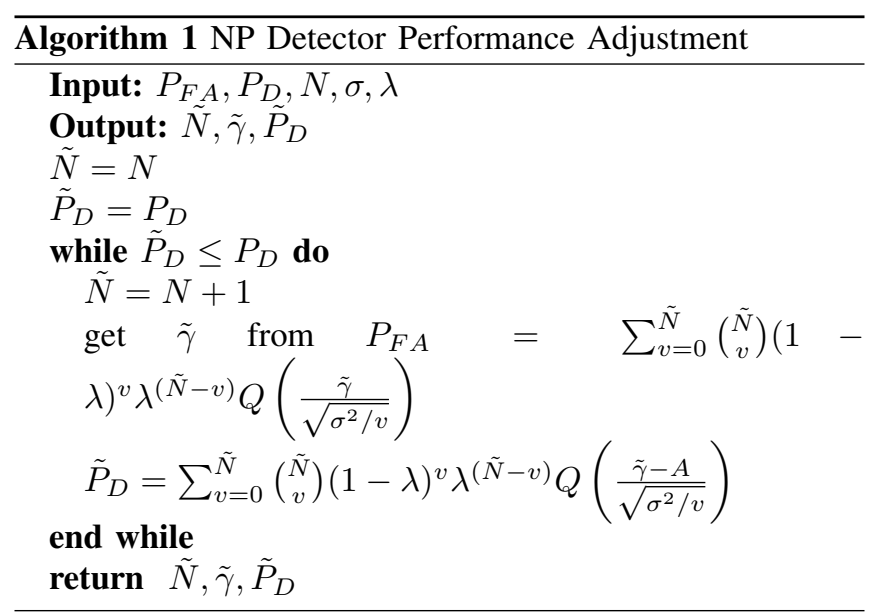

observations to $\tilde{N}$ that achieves the same original performance represented by $\left(P_{F A}, P_{D}\right)$, possibly with a different threshold $\tilde{\gamma}$. Algorithm 1 illustrates the steps to obtain $\tilde{N}$ and $\tilde{\gamma}$ to restore the original detector performance. The basic idea of the algorithm is to calculate iteratively the new number of samples, $\tilde{N}$, that achieves the original $P_{F A}$, with $\tilde{P}_{D} \geq P_{D}$. In doing so, for every value of $\tilde{N}$ we solve Equation (8) to get $\tilde{\gamma}$, and then substitute for $\tilde{\gamma}$ in Equation (9) to get $\tilde{P}_{D}$. The algorithm stops if $\tilde{P}_{D} \geq P_{D}$. Equation (8) is an implicit equation in $\tilde{\gamma}$, so it is solved iteratively with an initial guess. The initial guess of $\tilde{\gamma}=\gamma$ (original detector threshold) is found to speed up the convergence process.

Algorithm 1 is run on the detector with parameters listed in Table I $(\lambda=0.2)$, and the numerical results are listed in Table III.

TABLE III

NEW DETECTOR PARAMETERS

\begin{tabular}{|c||c|c|c|c|c|c|c|}
\hline$\tilde{N}$ & 51 & 52 & 53 & $\ldots$ & 61 & 62 & 63 \\
\hline$\tilde{P}_{D}$ & 0.3554 & 0.3600 & 0.3647 & $\ldots$ & 0.4010 & 0.4054 & 0.4098 \\
\hline$\tilde{\gamma}$ & 0.2581 & 0.2556 & 0.2532 & $\ldots$ & 0.2359 & 0.2340 & 0.2321 \\
\hline
\end{tabular}

An important result is that the increased number of samples, $\tilde{N}$, that can be systematically computed, restores the original performance for all values of $P_{F A}$. In other words, using $\tilde{N}$, the new ROC curve can be made to coincide with the original ROC curve. This fact is clearly shown in Figure 4, and this makes it possible to construct a Delay-Drop Rate curve for every detector design, defined by Equation (7). In the Delay-Drop Rate curve, the channel drop rate, $\lambda$, is plotted on the abscissa $(0 \leq \lambda \leq 1)$ and the corresponding extra number of samples required to restore the performance, $\tilde{N}$, is plotted on the ordinate. Figure 5 illustrates the DelayDrop Rate curve for the example detector given in this paper, with parameters as in Table I. It is shown that the number of samples approximately doubles with a drop rate $\lambda=0.5$, and the curve exhibits an exponential behavior thereafter.

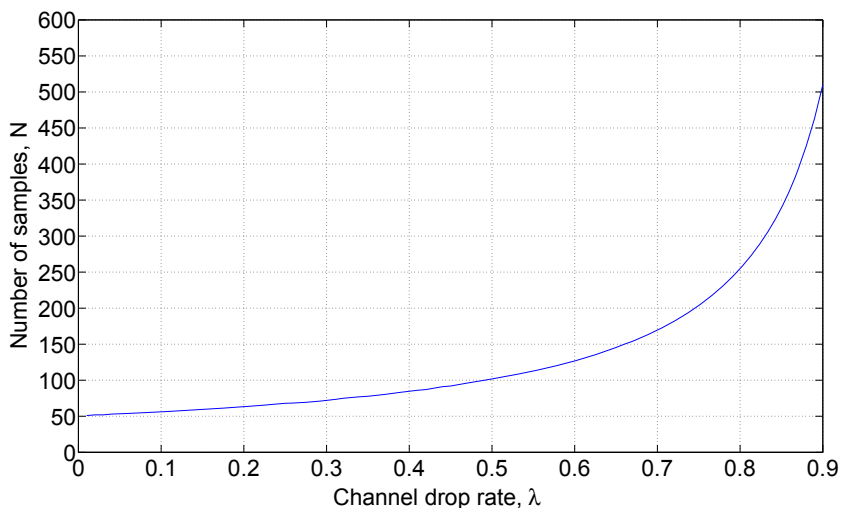

Fig. 5. Delay-Drop Rate curve

The Delay-Drop Rate curve can be used for adaptive detector design. This is best illustrated by Figure 1. The channel drop rate, $\lambda$, is estimated by statistical measurements, and using the Delay-Drop Rate curve the new number of samples, $\tilde{N}$ is obtained. The detector is then reconfigured online by changing the original number of samples, $N$, to the new value, $\tilde{N}$.

\section{CONCLUSIONS AND FUTURE WORK}

In this paper we have addressed the problem of Neyman Pearson detection with intermittent observations, modeled by a Bernoulli channel. The motivation for this problem is the emerging technology of passive wireless sensors and their deployment in problems where event detection is of a prime interest. We have studied the detection problem in a single sensor to reader configuration. We formulated the detection performance with intermittent observations modeled as a Bernoulli random process. We presented a case study for the problem of detecting a known DC level in WGN and showed the degradation in the performance in terms of the ROC curve. We have also presented an adaptive detector architecture, where a pre-constructed Delay-Drop Rate curve is used for detector reconfiguration.

The work in the paper presents the performance analysis of the detector, given that the detector is already designed, without considering the channel effect. We are currently working on the optimal detector design, taking into account the channel effect. Also, the channel is modeled by a memoryless, Bernoulli random process. In many practical applications, 
an error in the packet is usually caused by a bursty noise, which causes an error in a number of subsequent packets. This could be modeled, as an example, using a Markov chain with finite memory. We are currently studying the detection performance with such more sophisticated channel models.

Delay for detection is very important in a major class of detection problems. Therefore, we are currently investigating other possible solutions to restore the original performance without introducing excess delay in the detection process. Multiple sensors and data fusion are one of the solutions currently under study.

\section{REFERENCES}

[1] J.-F. Chamberland and V. V. Veeravalli, "Wireless sensors in distributed detection applications," IEEE Signal Processing Magazine, 2007.

[2] B. Chen, R. Jiang, T. Kasetkasem, and P. K. Varshney, "Channel aware decision fusion in wireless sensor networks," IEEE Transactions on Signal Processing, vol. 52, no. 12, pp. 3454-3458, Dec. 2004.

[3] B. Chen, L. Tong, and P. K. Varshney, "Channel-aware distributed detection in wireless sensor networks," IEEE Signal Processing Magazine, July 2006.

[4] B. Chen and P. K. Willett, "On the optimality of the likelihoodratio test for local sensor decision rules in the presence of nonideal channels," IEEE Transactions on Information Theory, vol. 51, no. 2, pp. 693-699, Feb. 2005.

[5] R. D. Hippenstiel, Detection Theory: Applications and Digital Signal Processing. Boca Raton, FL: CRC Press, 2002.

[6] S. M. Kay, Fundamentals of Statistical Signal Processing, Volume 2: Detection Theory, ser. Prentice Hall Signal Processing Series, A. V. Oppenheim, Ed. Prentice Hall PTR, 1998.

[7] S. Lewis, "A basic introduction to RFID technology and its use in the supply chain," LARAN RFID, Tech. Rep., 2004.

[8] P. Li and Y. Wen, "Design and fabrication of passive wireless sensor array system using composite coding resonant saw transducer." Measurement Science and Technology, Institute of Physics Publishing, UK, vol. 17, no. 2, 2006.
[9] R. Matsuzaki and A. Todoroki, "Passive wireless strain monitoring of actual tire using capacitance-resistance change and multiple spectral features," Sensors and Actuators A: Physical, Elsevier B.V., vol. 126, no. 2, 2005.

[10] T. K. Moon and W. C. Stirling, Mathematical Methods and Algorithms for Signal Processing. Prentice Hall, Upper Saddle River, NJ, 2000.

[11] B. Sinopoli, L. Schenato, M. Franceschetti, K. Poolla, M. Jordan, and S. Sastry, "Kalman filtering with intermittent observations," IEEE Transactions on Automatic Control, vol. 49, no. 9, pp. 1453-1464, Sept. 2004.

[12] A. Tantawy, X. Koutsoukos, and G. Biswas, "Composite hypothesis testing with intermittent observations for passive wireless sensors," 7th IFAC Symposium on Fault Detection, Supervision, and Safety of Technical Processes, 2009, to appear.

[13] _ _ "Maximum likelihood detection with intermittent observations," IEEE Conference on Information Sciences and Systems, 2009, to appear.

[14] R. R. Tenney and N. R. Sandell, "Detection with distributed sensors," IEEE Transactions on Aerospace and Electronic Systems, vol. AES17, no. 4, pp. 501-510, July 1981.

[15] S. C. A. Thomopoulos and L. Zhang, "Distributed decision fusion with networking delays and channel errors," Inform. Sci., vol. 66, pp. 91-118, 1992.

[16] F. Umbrecht, M. Wendlandt, D. Juncker, C. Hierold, and J. Neuenschwander, "A wireless implantable passive strain sensor system," IEEE Conference on Sensors, pp. 4 pp.-, Oct.-3 Nov. 2005.

[17] V. V. Veeravalli and J.-F. Chamberland, "Detection in sensor networks," in Wireless Sensor Networks: Signal Processing and Communications Perspectives, Y.-W. H. A. Swami, Q. Zhao and L. Tong, Eds. John Wiley \& Sons, Ltd, 2007, ch. 6, pp. 119-148.

[18] H. Yoon, J. Xie, J. Abraham, V. Varadan, and P. Ruffin, "Passive wireless sensors using electrical transition of carbon nanotube junctions in polymer matrix," Smart Materials and Structures, Institute of Physics Publishing, UK, vol. 15, no. 1, 2006.

[19] X. Zhang and F.-Y. Wang, "Key technologies of passive wireless sensor networks based on surface acoustic wave resonators," IEEE International Conference on Networking, Sensing and Control, ICNSC 2008, pp. 1253-1258, April 2008. 